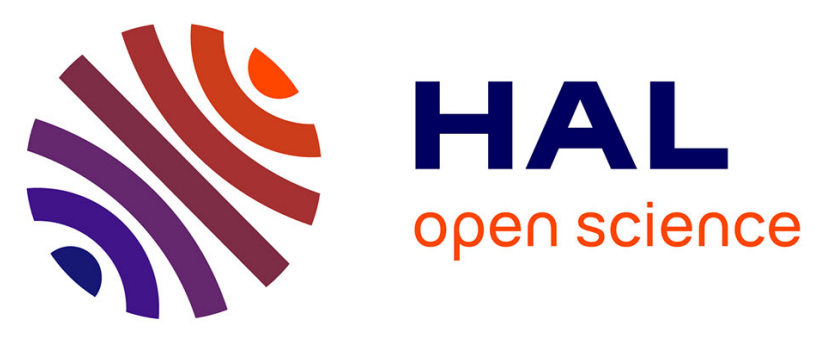

\title{
X-ray resonant magnetic reflectivity of stratified magnetic structures: Eigenwave formalism and application to a $\mathrm{W} / \mathrm{Fe} / \mathrm{W}$ trilayer
}

Marta Elzo, Emmanuelle Jal, Oana Bunau, Stéphane Grenier, Aline Y. Ramos, Hélio Tolentino, Yves Joly, Jean-Marc Tonnerre, Nicolas Jaouen

\section{To cite this version:}

Marta Elzo, Emmanuelle Jal, Oana Bunau, Stéphane Grenier, Aline Y. Ramos, et al.. X-ray resonant magnetic reflectivity of stratified magnetic structures: Eigenwave formalism and application to a W/Fe/W trilayer. Journal of Magnetism and Magnetic Materials, 2012, 324 (2), pp.105-112. 10.1016/j.jmmm.2011.07.019 . hal-00594121v2

HAL Id: hal-00594121

https://hal.science/hal-00594121v2

Submitted on 24 Apr 2012

HAL is a multi-disciplinary open access archive for the deposit and dissemination of scientific research documents, whether they are published or not. The documents may come from teaching and research institutions in France or abroad, or from public or private research centers.
L'archive ouverte pluridisciplinaire HAL, est destinée au dépôt et à la diffusion de documents scientifiques de niveau recherche, publiés ou non, émanant des établissements d'enseignement et de recherche français ou étrangers, des laboratoires publics ou privés. 


\title{
$\mathrm{X}$-ray resonant magnetic reflectivity of stratified magnetic structures: eigenwave formalism and application to a $\mathrm{W} / \mathrm{Fe} / \mathrm{W}$ trilayer
}

\author{
M. Elzo \\ Institut Néel, CNRSEUniversité Joseph Fourier, BP 166, F-38042, Grenoble Cedex 9, France and \\ Synchrotron SOLEIL, L'Orme des Merisiers, 91192 Gif/Yvette, France \\ E. Jal, O. Bunau, S. Grenier, Y. Joly, A. Y. Ramos, H. C. N. Tolentino, and J.M. Tonnerre \\ Institut Néel, CNRSGUniversité Joseph Fourier, BP 166, F-38042, Grenoble Cedex 9, France \\ N. Jaouen \\ Synchrotron SOLEIL, L'Orme des Merisiers, 91192 Gif/Yvette, France
}

(Dated: August 22, 2011)

\begin{abstract}
A formalism for the reflectivity of electromagnetic waves by magnetic materials is presented with an application. It is applied to retrieve the magnetic moment density along the depth of magnetic materials with arbitrary magnetic moment direction using matricial algebra, including roughness between layers. The reflectivity is derived following a classical description with Maxwell equations and a permittivity built from the quantum scattering amplitude. Approximations on the relative power of the Thomson scattering and the magnetic terms are trackable in order to evaluate the validity of the formalism case-by-case, from the optical light regime up to soft and hard X-rays. Eigenwaves are used throughout the whole formalism. In order to illustrate the methodology, we present an application to a $\mathrm{W} / \mathrm{Fe} / \mathrm{W}$ trilayer performed at the Fe $L$-edge, in the soft X-ray regime.
\end{abstract}

\section{INTRODUCTION}

X-ray resonant magnetic reflectivity (XRMR) yields the magnetization density across ultra-thin magnetic materials. Two specificities have established the technique as a complement to macroscopic techniques or polarized neutron scattering: a sensitivity to the orientation and the amplitude of the local magnetic moment with a spatial resolution below the nanometer, and the selection of the chemical element contributing to the magnetization. The sensitivity to magnetism is based on the Faraday effect [1], or equivalently on the magneto-optic Kerr effect (MOKE) [2, 3], that is, the rotation of the polarization of the optical photon due to the magnetization, respectively, in transmission or in reflectivity geometries. With the shorter wavelength of X-rays, and in reflectivity mode, one obtains the spatial resolution and the sensitivity to spatially dependent non-colinear magnetism, like canted or antiferromagnetism. XRMR selects each chemical species contributing to the magnetization thanks to the chemically specific resonant effect of the scattering with X-rays, as detailed hereafter. Moreover, such a photon in - photon out technique enables measurements on sample under applied electric or magnetic fields, allowing in operando characterization. The sensitivity of the resonant X-ray scattering to the magnetic moment amplitude is significant, the magnetic-dependent scattering length can be tens of $r_{0}$, the single electron's photon scattering length, as discovered by Gibbs et al. in Holmium in 1988 $[4]$.

In this paper, we present a formalism for resonant magnetic reflectivity applied to stratified magnetic media. We use a classical approach to calculate the propagation of the eigenwaves in the media, and a quantum de- scription of the atomic interaction with the photon. We show that by using eigenwaves throughout the treatment the formalism of the technique gains a relative simplicity compared to preceding, otherwise similar, formalisms presented in the literature; we also keep trackable the approximations in order to qualify their use. The formalism can be applied to retrieve magnetization profiles in magnetic media such as thin films or multilayers on substrates, with magnetization in any direction, whenever spin-orbit coupling can be neglected, either because of cubic symmetry or because of quenched angular momentum. It can also be applied to magneto-optics if the magnetic correction to the permittivity is relatively small. Finally, we present an application of the formalism with a detailed methodology to illustrate some of the specificities.

\section{EIGEN-WAVE FORMALISM}

\section{A. Generalities}

In the following sections we consider the classical approach by which the propagation of electro-magnetic waves in a medium is described by Maxwell equations. Assuming that the medium has a linear, tensorial and dispersive response to the electric field and a simple linear, scalar response to the magnetizing field, the constitutive relations considered here are:

$$
D_{i}=\epsilon_{i j} E_{j}, \mathbf{B}=\mu \mathbf{H}
$$

where $\epsilon_{i j}$ is a tensorial dielectric permittivity and $\mu$ the scalar magnetic permeability, $\mathbf{D}$ the electric displacement, $\mathbf{E}$ the electric field, $\mathbf{B}$ the magnetic field and $\mathbf{H}$ 
the magnetizing field. One further considers that the effect of the medium on the wave can essentially be reduced to a change in the wave vector, in direction and in amplitude, in addition to absorption. Solutions to the propagation equation are sought in the form of propagating waves with wave vector $\mathbf{k}$ :

$$
\mathbf{k}=\frac{2 \pi}{\lambda} \mathbf{n}
$$

The index of refraction $\mathbf{n}$ is imposed by the solution of Maxwell equations, it will depend on $\epsilon_{i j}$. To solve the problem entirely, boundary conditions are applied at interfaces as Maxwell equations impose conservations of the plane components of the exciting fields $\mathbf{E}$ and $\mathbf{H}$ at interfaces. The problem of reflectivity and transmission is therefore reduced to solving the conservation of the plane components at the interface followed by the propagation in the medium. The formalism leads to a recursive multiplication of matrices, as commonly used in optics.

\section{B. Near X-ray resonance susceptibilities}

\section{Dielectric Permittivity}

For a classical description and a historical account on the magnetic dependence of the scattering amplitude, one may refer to Qiu and Bader in Ref. [3]. In the following, we build the dielectric permittivity from the scattering amplitude obtained by the quantum mechanical treatment of the atomic scattering as presented by Hannon et al. $[5,6]$. The relation between the scattering factors and the dielectric permittivity is:

$$
\begin{aligned}
& \hat{\epsilon}=1+\hat{\chi} \\
& \hat{\chi}=\frac{4 \pi}{k_{0}^{2}} \sum_{a} \rho_{a} \hat{f}_{a}
\end{aligned}
$$

with $\rho_{a}$ the number of atoms $a$ per unit volume and $\hat{f}_{a}$ its scattering length. The scattering amplitude is developed as:

$$
\hat{f}_{\boldsymbol{e}^{\prime} \boldsymbol{e}}=\left(\boldsymbol{e}^{* *} \cdot \boldsymbol{e}\right) F_{0}-i\left(\boldsymbol{e}^{\prime *} \times \boldsymbol{e}\right) \cdot \boldsymbol{u} F_{1}
$$

with

$$
\begin{aligned}
& F_{0}=-r_{0} f_{0}+(3 / 4 k)\left[F_{11}+F_{1 \overline{1}}\right] \\
& F_{1}=(3 / 4 k)\left[F_{11}-F_{1 \overline{1}}\right]
\end{aligned}
$$

where the polarization vectors of the incoming and outgoing photons, $e$ and $e^{\prime}$ respectively, are unit vectors equivalent to the electric field vector of the classical description, $\boldsymbol{e}=\boldsymbol{E} / E . \quad r_{0} f_{0}$ is the Thomson term, $r_{0}$ is the classical electron radius and $f_{0}$ the spatial Fourier transform of the electron charge density. The $F_{1 m}$ functions are proportional to the probability of absorption of a photon by an atom with a change of $m$ in its atomic angular momentum projected along $\boldsymbol{u}$. The unit vector $\boldsymbol{u}$ is the quantification axis, here it is chosen to correspond to the direction of the local magnetic moment. The term $F_{1}$ contains the magnetic signal, which lies in the difference $F_{11}-F_{1 \overline{1}}$ corresponding to the difference in absorption with the angular momentum change $\Delta m= \pm 1$. Expression (4) corresponds to an atom whose electronic configuration, including the spin, can be described in a point group symmetry as low as $C_{4 h}$, which is enough to describe an uniaxial asymmetry defined by the unit vector $\boldsymbol{u}$. It typically describes a cubic symmetry broken by a magnetic moment $\boldsymbol{u}=\boldsymbol{m} / \mathrm{m}$. We neglect a spinorbit interaction in the valence shell that would distort the spatial configuration of orbitals and lower the symmetry. A significant spin-orbit coupling in the valence shells would give rise to linear magnetic dichroism; such cases of lower symmetries are considered for the atomic scattering factor in two papers $[7,8]$. Here, the local cubic symmetry is only broken by the magnetic moment. Finally, the expression (4) also implies that we restrict ourselves to the approximation of a dipolar transition, that is $\Delta l=1$ and $\Delta m=0, \pm 1$; higher orders in the multipolar expansion are neglected. This approximation corresponds to neglecting the phase change of the propagating photon inside the orbitals involved in the photoelectric transition, i.e. $r / \lambda \ll 1$. Such approximation is appropriate for soft X-rays where $\lambda$ is of the order of $100 \bar{r}, \bar{r}$ being the average distance of the $2 p$ electron from the nucleus, $0.07 \AA$ for $\mathrm{Fe}$ (if there were any magnetized density in $f$ states). However, for hard X-rays one may expect some contribution of quadrupolar transition, $l=2$, even though the terms are still much smaller than those for the dipolar transitions. In the following, we do not include the terms of higher order.

Expression (4) makes explicit the dependence of the scattering length with the incoming and the outgoing polarization. Let's rewrite this expression in an equivalent form by referring explicitly to the spatial directions as in Fig. 1. We have $\hat{f}_{\boldsymbol{e}^{\prime} \boldsymbol{e}}=\boldsymbol{e}^{\prime *} \cdot \hat{f} \cdot \boldsymbol{e}$ with:

$$
\hat{f}=\left(\begin{array}{rrr}
F_{0} & -i u_{z} F_{1} & i u_{y} F_{1} \\
i u_{z} F_{1} & F_{0} & -i u_{x} F_{1} \\
-i u_{y} F_{1} & i u_{x} F_{1} & F_{0}
\end{array}\right)
$$

From the expressions (3) and (4), one derives the expression of the element specific dielectric tensor:

$$
\hat{\epsilon}=\left(\begin{array}{ccc}
\epsilon & \epsilon_{x y} & \epsilon_{x z} \\
-\epsilon_{x y} & \epsilon & \epsilon_{y z} \\
-\epsilon_{x z} & -\epsilon_{y z} & \epsilon
\end{array}\right)
$$

that appears antisymmetric, and whose diagonal terms, all equal, and off-diagonal terms are:

$$
\begin{aligned}
\epsilon & =1+\frac{4 \pi}{k_{0}^{2}} \rho F_{0} \\
\epsilon_{x y} & =-i \frac{4 \pi}{k_{0}^{2}} \rho u_{z} F_{1} \\
\epsilon_{x z} & =i \frac{4 \pi}{k_{0}^{2}} \rho u_{y} F_{1}
\end{aligned}
$$




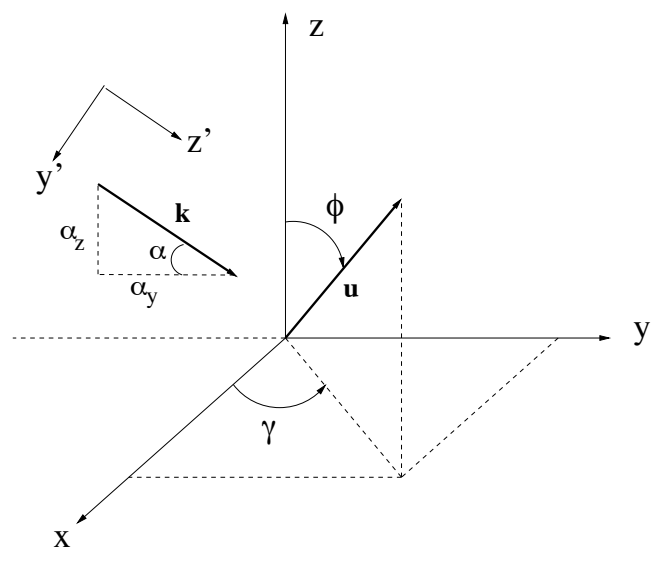

FIG. 1. Referential and definition of angles. Here, $\hat{\mathbf{k}}$ is a unit vector, $\alpha_{y}=\cos \alpha$ and $\alpha_{z}=\sin \alpha$. The referential $\left(x^{\prime}, y^{\prime}, z^{\prime}\right)$ is attached to the wave vector, with $x^{\prime}=x$ and $\hat{\boldsymbol{k}}=\hat{\boldsymbol{z}}^{\prime}$.

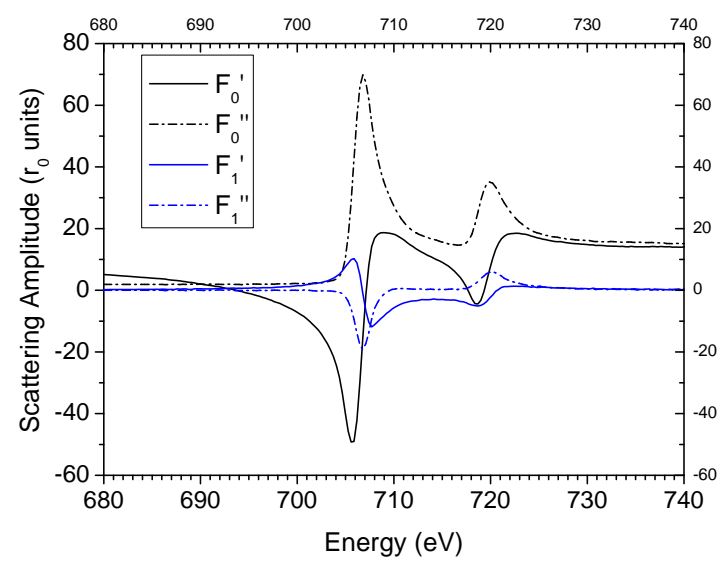

FIG. 2. Real (') and imaginary (") parts of the scattering length (in $r_{0}$ unit) at the $\mathrm{Fe} \mathrm{L}_{3,2}$ edge.

$$
\epsilon_{y z}=-i \frac{4 \pi}{k_{0}^{2}} \rho u_{x} F_{1}
$$

An example of real and imaginary parts of $F_{0}$ and $F_{1}$ for $\mathrm{Fe}$ atoms are shown in Fig. 2. These factors were obtained after experimental X-ray absorption measurements of X-ray magnetic circular dichroism that provide the imaginary part and Kramers-Kronig transform of the imaginary part to give the real part [9]. The dielectric permittivity components are shown in Fig. 3 and 4. This dielectric permittivity is then injected into Maxwell's classical equations, and the related equation of wave propagation.

\section{Magnetic permeability}

We assume that the magnetic origin of the scattering is purely electric in nature, due to spin-orbit effects in the core-hole at the resonance. We will thus assume $\mu=1$.

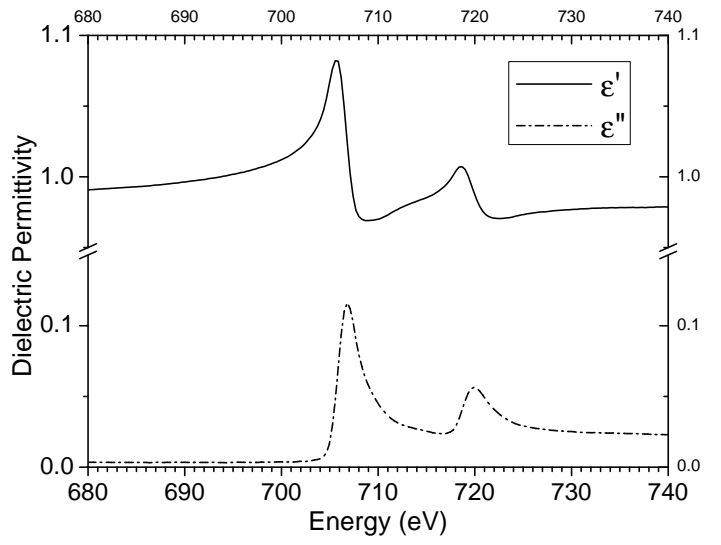

FIG. 3. Real and imaginary parts of the magneticindependent dielectric tensor elements of Fe atoms near the $\mathrm{L}_{3,2}$ edge.

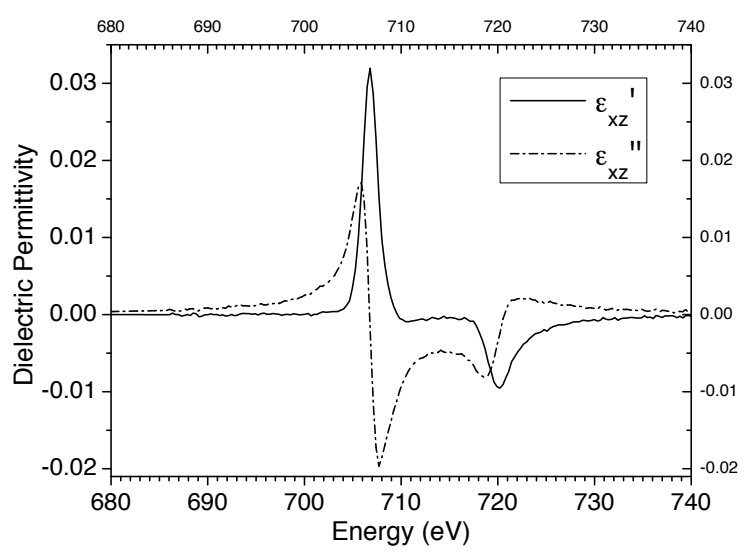

FIG. 4. Real and imaginary parts of the magnetic-dependent dielectric tensor elements of $\mathrm{Fe}$ atoms near the $\mathrm{L}_{3,2}$ edge.

\section{Solutions to Maxwell equations}

\section{Fresnel equation and index of refraction}

Before deriving the expression of the reflectivity we must find how the waves propagate in a medium (how the phase of the wave evolves) so as to determine the eigenwaves for which the polarization is unchanged as they propagate within one medium. Maxwell equations lead to a propagation equation (Helmholtz equation) for which one seeks a monochromatic wave with wave vector $\mathbf{k}=\frac{2 \pi}{\lambda} \mathbf{n}$ as a solution. This procedure leads to $\mathbf{n} \times(\mathbf{n} \times$ $\boldsymbol{E})+\hat{\epsilon} \boldsymbol{E}=0$, which constrains the indices of refraction with respect to the permittivity [10]:

$$
\left|n^{2} \delta_{i k}-n_{i} n_{k}-\epsilon_{i k}\right|=0
$$


Considering a referential attached to a wave propagating along $z^{\prime}, \mathbf{n}=n(0,0,1)$, the solutions are:

$$
n^{2}=\epsilon+\frac{\epsilon_{x^{\prime} z^{\prime}}^{2}+\epsilon_{y^{\prime} z^{\prime}}^{2} \pm \sqrt{\left(\epsilon_{x^{\prime} z^{\prime}}^{2}+\epsilon_{y^{\prime} z^{\prime}}^{2}\right)^{2}-4 \epsilon^{2} \epsilon_{x^{\prime} y^{\prime}}^{2}}}{2 \epsilon}
$$

The expression for $n$ has four solutions corresponding to four waves, two pairs going in opposite directions, with two polarization states for each pair.

\section{Small magnetic-dependent dielectric terms approximation}

From now on, we limit our approach to the approximation $\epsilon_{i j}^{2} \ll \epsilon$, neglecting the second order of the offdiagonal terms of the permittivity. This approximation greatly simplifies the relations; its justification is empirical and theoretical as obtained from $a b$ initio calculations, but it should be checked for each case. One can observe that this approximation is totally justified for soft X-rays. Even if the magnetic-dependent scattering amplitude is of the same order as $r_{0}$, the most important term is the first order term. The index of refraction becomes:

$$
n \approx \sqrt{\epsilon \pm i \epsilon_{x^{\prime} y^{\prime}}}
$$

and developing the permittivity:

$$
n^{ \pm}=\sqrt{1+\frac{4 \pi}{k_{0}^{2}} \rho\left(F_{0} \pm u_{z^{\prime}} F_{1}\right)} \approx 1+\frac{2 \pi}{k_{0}^{2}} \rho\left(F_{0} \pm u_{z^{\prime}} F_{1}\right) .
$$

In this approximation, the index of refraction only depends on the component of the magnetic moment that is along the propagation vector. Generalizing to an arbitrary referential, the index of refraction is:

$n^{ \pm}=\sqrt{1+\frac{4 \pi}{k_{0}^{2}} \rho\left(F_{0} \pm \hat{\boldsymbol{k}} \cdot \boldsymbol{u} F_{1}\right)} \approx 1+\frac{2 \pi}{k_{0}^{2}} \rho\left(F_{0} \pm \hat{\boldsymbol{k}} \cdot \boldsymbol{u} F_{1}\right)$

The planar component of the wave vector is conserved (Snell's law), with $n_{0}$ an isotropic medium (usually vacuum with $n_{0}=1$ ):

$$
n_{0} \alpha_{y 0}=n^{+} \alpha_{y}^{+}=n^{-} \alpha_{y}^{-}
$$

That is, in magnetic media, the wave vectors of the two eigenwaves generally have different directions unless the local magnetization $\boldsymbol{u}$ is perpendicular to the propagation vector.

\section{Polarization of the eigenwaves}

To find the polarization states corresponding to the indices in expression (10), one combines Maxwell equations, and gets the expression $\mathbf{D}=\mathbf{n} \times(\mathbf{E} \times \mathbf{n})$ which leads to:

$$
\begin{aligned}
& D_{x^{\prime}}=\left(\epsilon \pm i \epsilon_{x^{\prime} y^{\prime}}\right) E_{x^{\prime}} \\
& D_{y^{\prime}}=\left(\epsilon \pm i \epsilon_{x^{\prime} y^{\prime}}\right) E_{y^{\prime}} \\
& D_{z^{\prime}}=0
\end{aligned}
$$

Equating with the constitutive equation, $D_{i}=\epsilon_{i j} E_{j}$, one finds the ratio:

$$
D_{y^{\prime}} / D_{x^{\prime}} \approx \pm i
$$

associated with

$$
n^{ \pm}=\sqrt{\epsilon \pm i \epsilon_{x^{\prime} y^{\prime}}}
$$

having again neglected cross-products of the off-diagonal terms of the permittivity. The eigenwaves propagating in a magnetic medium with the permittivity as in Eq. (7) are circularly polarized waves only if we determine that higher order of the off-diagonal terms can be neglected. In that same reference frame, the circular waves have for general components:

$$
\mathbf{D}^{ \pm}=\mp \frac{D_{x^{\prime}}^{ \pm}}{\sqrt{2}}\left(\begin{array}{c}
1 \\
\pm i \\
0
\end{array}\right)
$$

The overall $\mp$ sign stands for the convention by which the angular momentum is $\pm \hbar$ along the propagation. In the referential depicted in Fig. 1, the components of the waves become:

$$
\mathbf{D}^{ \pm}=\frac{D_{x}^{ \pm}}{\sqrt{2}}\left(\begin{array}{c}
\mp 1 \\
i \alpha_{z}^{ \pm} \\
i \alpha_{y}^{ \pm}
\end{array}\right)
$$

At this point, one has determined the waves that propagate in a medium with a dielectric tensor having small antisymmetric off-diagonal terms, and constant diagonal terms. The propagation through an interface is described by applying Maxwell equations at the boundary.

\section{Boundary conditions}

Maxwell equations impose boundary conditions to planar components of the electromagnetic fields $\mathbf{E}$ and $\mathbf{H}$ which must be continuous through the interface. One is led to find these components for the eigenwaves that were derived in the previous section. Considering the eigenwaves $D^{ \pm}$, the corresponding exciting fields are:

$$
\begin{array}{r}
\mathbf{E}=\hat{\epsilon}^{-1} \mathbf{D} \\
\mathbf{H}=\mathbf{n} \times \mathbf{E}
\end{array}
$$

The inverse of the antisymmetric dielectric tensor,

$$
\hat{\epsilon}^{-1}=\frac{1}{|\epsilon|}\left[\begin{array}{ccc}
1+\epsilon_{y z}^{2} & -\epsilon_{x y}-\epsilon_{x z} \epsilon_{y z} & -\epsilon_{x y}+\epsilon_{x y} \epsilon_{y z} \\
\epsilon_{x y}-\epsilon_{x z} \epsilon_{y z} & 1+\epsilon_{x z}^{2} & -\epsilon_{y z}-\epsilon_{x y} \epsilon_{x z} \\
\epsilon_{x y}+\epsilon_{x y} \epsilon_{y z} & +\epsilon_{y z}-\epsilon_{x y} \epsilon_{x z} & 1+\epsilon_{x y}^{2}
\end{array}\right]
$$


can be approximated by neglecting again second order terms in $\epsilon_{i j}$ :

$$
\hat{\epsilon}^{-1} \approx \frac{1}{\epsilon}\left[\begin{array}{ccc}
1 & -\epsilon_{x y} & -\epsilon_{x z} \\
\epsilon_{x y} & 1 & -\epsilon_{y z} \\
\epsilon_{x z} & \epsilon_{y z} & 1
\end{array}\right]
$$

Relations (17) and (20), with (18), lead to:

$$
\begin{aligned}
& E_{x}^{ \pm}=D_{x}^{ \pm} \frac{1}{\sqrt{2} \epsilon}\left(\mp 1-i \epsilon_{x y} \alpha_{z}^{ \pm}-i \epsilon_{x z} \alpha_{y}^{ \pm}\right) \\
& E_{y}^{ \pm}=D_{x}^{ \pm} \frac{1}{\sqrt{2} \epsilon}\left(\mp \epsilon_{x y}+i \alpha_{z}^{ \pm}-i \epsilon_{y z} \alpha_{y}^{ \pm}\right) \\
& E_{z}^{ \pm}=D_{x}^{ \pm} \frac{1}{\sqrt{2} \epsilon}\left(\mp \epsilon_{x z}+i \epsilon_{y z} \alpha_{z}^{ \pm}+i \alpha_{y}^{ \pm}\right)
\end{aligned}
$$

and, with relation (19), lead to:

$$
\begin{aligned}
& H_{x}^{ \pm}=D_{x}^{ \pm} \frac{1}{\sqrt{2} \epsilon} n^{ \pm}\left(i \mp \epsilon_{x y} \alpha_{z}^{ \pm} \mp \epsilon_{x z} \alpha_{y}^{ \pm}\right) \\
& H_{y}^{ \pm}=D_{x}^{ \pm} \frac{1}{\sqrt{2} \epsilon} n^{ \pm} \alpha_{z}^{ \pm}\left( \pm 1+i \epsilon_{x y} \alpha_{z}^{ \pm}+i \epsilon_{x z} \alpha_{y}^{ \pm}\right)
\end{aligned}
$$

With a close look at the resulting expressions one can simplify the writing of the magnetic field's planar components to $H_{x}^{ \pm}=\mp i n^{ \pm} E_{x}^{ \pm}$and $H_{y}^{ \pm}=-\alpha_{z}^{ \pm} n^{ \pm} E_{x}^{ \pm}$. We can now build the boundary matrix for the medium $m$. This matrix projects the state of the polarization of the wave into the planar components of the electric and magnetic field. The boundary matrix $A_{m}$ is then:

$$
\begin{gathered}
A_{m}=\frac{1}{\epsilon \sqrt{2}} \times \\
{\left[\begin{array}{cccc}
A_{x}^{+\downarrow} & A_{x}^{-\downarrow} & A_{x}^{+\uparrow} & A_{x}^{-\uparrow} \\
A_{y}^{+\downarrow} & A_{y}^{-\downarrow} & A_{y}^{+\uparrow} & A_{y}^{-\uparrow} \\
-i n^{+\downarrow} A_{x}^{+\downarrow} & i n^{-\downarrow} A_{x}^{-\downarrow} & -i n^{+\uparrow} A_{x}^{+\uparrow} & i n^{-\uparrow} A_{x}^{-\uparrow} \\
-\alpha_{z}^{+} n^{+\downarrow} A_{x}^{+\downarrow} & -\alpha_{z}^{-} n^{-\downarrow} A_{x}^{-\downarrow} & \alpha_{z}^{+} n^{+\uparrow} A_{x}^{+\uparrow} & \alpha_{z}^{-} n^{-\uparrow} A_{x}^{-\uparrow}
\end{array}\right]}
\end{gathered}
$$

where

$$
\begin{aligned}
A_{x}^{ \pm \downarrow} & =\mp 1-i \epsilon_{x y} \alpha_{z}^{ \pm}-i \epsilon_{x z} \alpha_{y}^{ \pm} \\
A_{x}^{ \pm \uparrow} & =\mp 1+i \epsilon_{x y} \alpha_{z}^{ \pm}-i \epsilon_{x z} \alpha_{y}^{ \pm} \\
A_{y}^{ \pm \downarrow} & =\mp \epsilon_{x y}+i \alpha_{z}^{ \pm}-i \epsilon_{y z} \alpha_{y}^{ \pm} \\
A_{y}^{ \pm \uparrow} & =\mp \epsilon_{x y}-i \alpha_{z}^{ \pm}-i \epsilon_{y z} \alpha_{y}^{ \pm}
\end{aligned}
$$

The arrows refer to the incident waves and propagates down $(-z)$ toward the substrate, and waves that propagate upward back to the surface and outgoing of the system. The third and fourth columns are the same as the two first, only $\alpha_{z}$ is changed to $-\alpha_{z}$. The four indices of refraction are given in eq. 13 .

In the case of an isotropic non-magnetic medium, all off-diagonal components of the dielectric tensor of the medium are null. The circular waves have the same indices, and the same angles. The boundary matrix for a non-magnetic medium reads:

$$
A=\frac{1}{n^{2} \sqrt{2}}\left[\begin{array}{cccc}
-1 & 1 & -1 & 1 \\
i \alpha_{z} & i \alpha_{z} & -i \alpha_{z} & -i \alpha_{z} \\
i n & i n & i n & i n \\
n \alpha_{z} & -n \alpha_{z} & -n \alpha_{z} & n \alpha_{z}
\end{array}\right]
$$

Finally the boundary conditions corresponding to equal planar components of the electromagnetic field can be expressed as:

$$
A_{m+1} D_{m+1}=A_{m} D_{m}
$$

which gives the polarization state of the downgoing and upgoing waves at the interface in the two media, for instance $D_{m+1}=A_{m+1}^{-1} A_{m} D_{m}$ in the $(m+1)$-th medium, at the interface with the $m$-th medium (see Fig. 5).

\section{E. Propagation}

While propagating within the medium, the electromagnetic waves undergo a change in the phase, $\phi=n \alpha_{z} z$, with the index of refraction $n$ and $\alpha_{z}$ corresponding to each of the four waves (see Fig. 1), $z$ being the depth position along the normal or the growth axis. With $D^{ \pm}$ as eigenwaves, the matrix $P$ for their propagation in the medium $m$ is:

$$
P_{m}(z)=\left[\begin{array}{cccc}
e^{-i \phi^{+\downarrow}} & 0 & 0 & 0 \\
0 & e^{-i \phi^{-\downarrow}} & 0 & 0 \\
0 & 0 & e^{i \phi^{+\uparrow}} & 0 \\
0 & 0 & 0 & e^{i \phi^{-\uparrow}}
\end{array}\right]
$$

The propagation matrix has its simplest form when eigenwaves are chosen for the basis. Absorption is taken into account by the complex index of refraction, that comes from the imaginary part of the scattering amplitude.

\section{F. Boundary-Propagation matrices}

In case of a multilayer, one multiplies the matrices corresponding to each medium. The final polarization state is:

$$
\begin{aligned}
D_{f} & =M D_{i}=A_{f}^{-1} \prod_{m} S_{m} A_{0} D_{i} \\
S_{m} & =A_{m} P_{m} A_{m}^{-1} \\
D_{f} & =\left[\begin{array}{c}
t_{C^{+}} \\
t_{C^{-}} \\
0 \\
0
\end{array}\right], D_{i}=\left[\begin{array}{c}
C_{i}^{+} \\
C_{i}^{-} \\
r_{C^{+}} \\
r_{C^{-}}
\end{array}\right]
\end{aligned}
$$

The matrix $\mathrm{M}$ is a $4 \times 4$ matrix which relates the polarization states of the incident and reflected waves with the waves transmitted in the last medium, defined as the medium in which there are no returning waves, like an homogeneous infinite substrate or vacuum. The matrices $S_{m}$ propagate the electric and magnetic fields in medium $m$ from one interface to the next interface. The polarization state of the initial incident waves, $C_{i}^{+}$and $C_{i}^{-}$, are the initial conditions, the problem is left with four unknowns and four equations. 


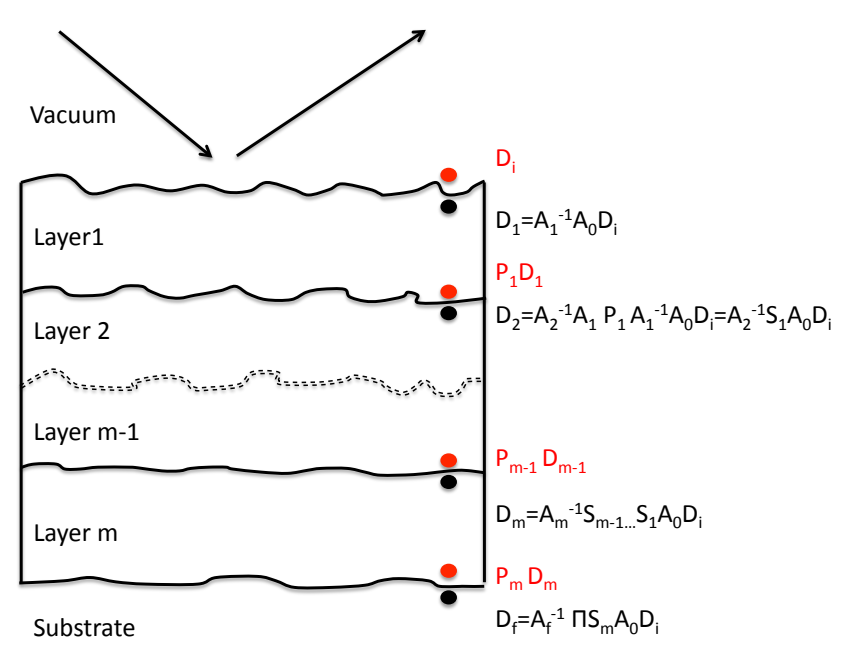

FIG. 5. Scheme indicating the polarization of the waves at each interfaces. $D_{i}=E_{i}$ is the polarization state of the electromagnetic field in the vacuum; $A_{m}^{-1} A_{m-1}$ relates the polarization states on both sides of an interface. $P_{m}$ propagates the wave between the two interfaces of the $m$-th layer.

\section{G. Reflectivity}

Reflectivity for both polarizations is readily obtained by solving the following system with known incident polarization amplitude, $C_{i}^{+}$and $C_{i}^{-}$:

$$
\left[\begin{array}{c}
t_{C^{+}} \\
t_{C^{-}} \\
0 \\
0
\end{array}\right]=M\left[\begin{array}{c}
C_{i}^{+} \\
C_{i}^{-} \\
r_{C^{+}} \\
r_{C^{-}}
\end{array}\right]
$$

The reflectivity can then be expressed as a matrix derived from the $\mathrm{M}$ matrix:

$$
\begin{aligned}
& {\left[\begin{array}{l}
r_{C^{+}} \\
r_{C^{-}}
\end{array}\right]=} \\
& {\left[\begin{array}{ll}
r_{C^{+} C^{+}} & r_{C^{+} C^{-}} \\
r_{C^{-} C^{+}} & r_{C^{-} C^{-}}
\end{array}\right]\left[\begin{array}{l}
C_{i}^{+} \\
C_{i}^{-}
\end{array}\right]} \\
& =\frac{1}{d} \times \\
& {\left[\begin{array}{ll}
M_{41} M_{34}-M_{31} M_{44} & M_{34} M_{42}-M_{32} M_{44} \\
M_{43} M_{31}-M_{41} M_{33} & M_{32} M_{43}-M_{33} M_{42}
\end{array}\right]\left[\begin{array}{c}
C_{i}^{+} \\
C_{i}^{-}
\end{array}\right]}
\end{aligned}
$$

with $d=M_{44} M_{33}-M_{34} M_{43}$.

If needed, consider the linear polarizations $\sigma$ and $\pi$, at this final step only, by inserting in the expression (30) the $2 \times 2$ matrices corresponding to the change of polarization basis from circular to linear polarizations: $C^{ \pm}=\mp 1 / \sqrt{2}(\sigma \pm i \pi)$. Ellipticity and Kerr rotation, that is the amount of rotated light, e.g. $r_{\pi \sigma} / r_{\sigma \sigma}$ for $\sigma$-incident light, can then be calculated. At third generation synchrotrons, the change of polarization between circular and linear polarizations is now commonly performed, and the use of circular polarization may be more usual.

\section{H. Roughness}

For the treatment of the roughness at the interface of the $(m-1)$-th with the $m$-th layer, the matrix element of $A_{m}^{-1} A_{m-1}$, which transform the amplitude of the circular waves from one interface to the next, must be multiplied one-by-one by $e^{ \pm}=e^{-\left(k_{m} \pm k_{m+1}\right)^{2} \sigma^{2} / 2}$ [11] with the following correspondence:

$$
W_{m, m+1}=\left[\begin{array}{llll}
e^{-} & e^{-} & e^{+} & e^{+} \\
e^{-} & e^{-} & e^{+} & e^{+} \\
e^{+} & e^{+} & e^{-} & e^{-} \\
e^{+} & e^{+} & e^{-} & e^{-}
\end{array}\right]
$$

$W$ being a table whose elements are multiplied by the corresponding element of the matrix $A_{m}^{-1} A_{m-1}$. Associated with this type of correction is the assumption that the roughness is Gaussian of width $\sigma$, in other words, the thickness is randomly distributed and that the dielectric tensor may be represented by an error function at the interface, $\epsilon=\epsilon_{m}+\left(\epsilon_{m-1}-\epsilon_{m}\right) \operatorname{Erf}(z, \sigma)$ with $\left.\operatorname{Erf}(z, \sigma)=(\sigma \sqrt{2 \pi})^{-1} \int_{-\infty}^{z} \exp \left(-\zeta^{2} / 2 \sigma^{2}\right) d \zeta\right)$. The roughness is also assumed to be stationary, that is, not dependent on the point under consideration. Note that following the work of Vidal and Vincent (Ref. [12]), the attenuation of the reflectivity does not depend on the basis on which the polarization of the wave is described, this table would thus be the same for a $\sigma$ and $\pi$ basis. A thorough description of magnetic and non-magnetic roughness is offered by Lee et al. in Ref. [13] and by Valencia in Ref. [14].

\section{DISCUSSION}

The present formalism is applicable to magneto-optics (for instance for Faraday effects, or MOKE simulations), as well as X-ray reflectivity on resonance or off resonance. Equivalent formalisms were presented by Mansuripur [15], Zak et al. [16], Bourzami et al. [17], and Qiu [3]. These formalisms are restricted to the antisymmetric dielectric tensor, as in the present paper. Several approximations are added for not using eigenwaves throughout the formalism. For instance, the projection of circular waves into a linear basis within a magnetic layer required approximations on the direction of the waves in order to calculate the electric field. The approximations are observed to not be important for the cases we have been dealing with, it is however an unnecessary step within these formalisms. Apart from the approximation made on the homogeneity of the media inherent to choosing macroscopic Maxwell equations, the main approximation 
of the present formalism lies in the relative value of offdiagonal terms of the dielectric tensor. We can see in the example of a high-spin atom like $\mathrm{Fe}$ in the bulk $b c c$ structure, in figure 4, that these terms may be at best in a ratio of 1:7 relative to the diagonal terms. One can also cite a seminal paper by Sacchi et al. who had presented results using a numerical approach to resolving Fresnel equation [18].

The formalisms presented by Stepanov et al. in Ref. [19] and Lee et al. [13] go to the next term of the development of the scattering amplitude $\left(F 2 \propto 2 F_{10}-F_{11}-F_{1 \overline{1}}\right)$, thereby including the possibility of electronic anisotropy in the valence shell, due to spin-orbit. These formalisms must be used in such specific cases where there is a strong spin-orbit coupling in the valence shell. This effect is, however, often absent or neglected in $3 d$ metallic or oxide materials, because of a high symmetry point group and quenched orbital momentum.

\section{METHODOLOGY AND EXAMPLE}

\section{A. Methodology}

XRMR has been applied to several types of thin films, multilayers and superlattices to determine the distribution of the magnetic moment density along the growth axis. The technique has proven (i) to be efficient on single magnetic layer in the nanometer thickness range [20, 21], (ii) to have a spatial resolution in the subnanometer range [22-24] and (iii) to be sensitive to small modifications at antiferromagnetic interfaces [25]. Induced magnetization of non magnetic elements has also been investigated [26]. The profile is averaged in both directions parallel to the plane but with a resolution about the Angström along the depth of the films. The sensitivity of resonant X-rays to all three components of the local magnetic moment has been exploited in various cases. For instance, Tonnerre et al. reported on the sensitivity to the moment normal to the surface, a timely application for the growing field of perpendicular magnets [23]. Spin-polarized neutron reflectivity is usually not preferable for lack of sensitivity to the moment's component along the scattering vector that is normal to the surface in such geometry. The authors also pointed out a key specificity of the XRMR technique with soft X-rays $(100 \mathrm{eV}$ to $1 \mathrm{keV})$. That is, soft X-ray reflectivity greatly eases a three-component "vectometric" measurement of the moment direction and amplitude. The component of the magnetic moment perpendicular to the surface is better probed when the angle of reflection is higher, whereas the other two components are better probed, from small angle up to near 45 degrees (the relations can be derived directly from the expression (4), and are given in Ref. [27]). It is a geometrical effect related to the relative orientation of the moment with the photon polarization. The phenomena of reflectivity is generally damped as $Q^{-4}$ [28], but $Q$ is still relatively small in the soft X-ray range and the reflectivity is measurable at a high angle, up to near back-scattering using an intense synchrotron X-ray source. Typically, with resonant hard X-rays, reflectivity could only be measured up to a mere 10 degrees for similar $Q$, therefore with poor sensitivity to the normal component of the magnetic moment. It is noteworthy that magnetic-sensitive edges of all $3 d$ metals are in the soft X-ray range.

The quantitative analysis of the magnetic properties is obtained after the refinement of energy and angle dependent resonant X-ray reflectivity collected in the vicinity of the magnetic-sensitive edges, like $L_{2,3}$ for $3 d$ metals. The magnetic signal is extracted from two successive measurements of the reflectivity corresponding to reversing either the direction of the magnetization or the photon angular moment. There is a choice for two experimental configurations. For one configuration, one specific photon polarization is chosen, being right, left circular or $\sigma$ and $\pi$ linear, and the magnetic field is reversed between two measurements. For the second configuration, the applied field is fixed, and the reflectivity is successively measured with the left and right circular polarizations. It is also possible, as in a XMLD experiment, to measure the contrast induced by two perpendicular incident linear polarizations of the photon. Note that the two configurations are generally equivalent, but not always, in particular when the magnetization exhibits a transverse magnetic component. The direction of the applied magnetic field is chosen according to the properties of the magnetic hysteresis, for instance along an easy magnetization axis. Once the configuration is chosen, the reflectivity measurement can be taken against the angle of incidence at one specific energy, as usual, or against the energy of the incident photon at one specific angle, or one specific momentum transfer. The choice of the energy is evident as the sensitivity to the magnetization is more pronounced at the edges. Usually a value close to the maximum of the absorption (where the transitions to polarized states may be more probable) is selected. It can be very useful to perform the measurements at the first inflection point (below the edge) to benefit from a strong variation of the real part of the magnetic terms of the resonant atomic scattering factor while avoiding strong absorption, and also at the second one (above the edge) where the effects previously mentioned are not as strong but where the variations of the resonant parameters can be sufficiently different (amplitude and sign) to produce very different signals. That feature can be very useful to move towards a unique model in case of a complex magnetic configuration. The choice of the angles depends on the sensitivity to a particular effect. For instance, a low angle will favor a refinement of a rather averaged magnetization because the reflectivity at low $Q$ value is less sensitive to the interferences between the layers and then not sensitive to the details of the variation of the amplitude of the magnetic moment across a layer. Measurements are usually made on maxima of fringes, related to a specific layer (when such an attribution is 


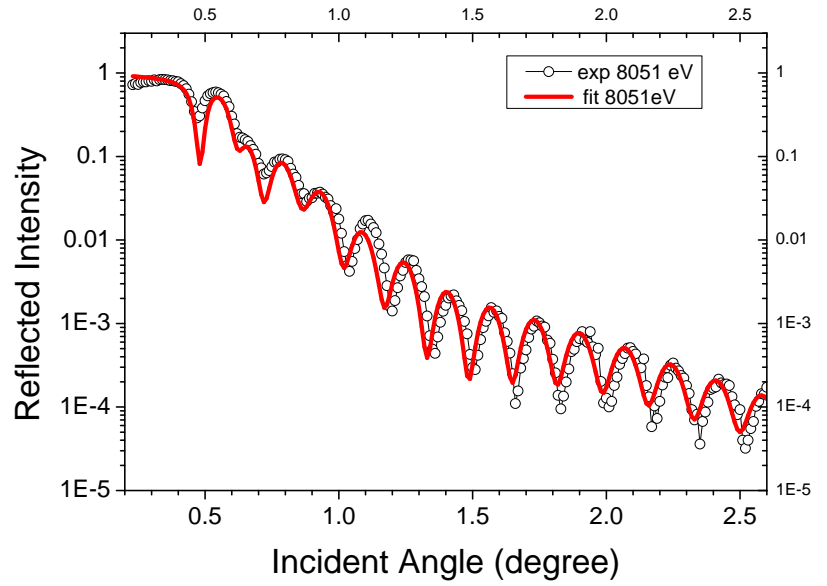

FIG. 6. Experimental (symbols) and calculated (solid line) specular reflectivity for $\mathrm{WFeW}$ at $8051 \mathrm{eV}$.

possible), on a set of angles that spans the angle range available with a significant intensity remaining for good statistics.

It is the angular or energy dependence of the extracted magnetic signal, the asymmetry, $R=\left(I^{+}-I^{-}\right) /\left(I^{+}+I^{-}\right)$ that is preferably refined. For energy dependent measurements, the difference, instead of the ratio, is preferred. For angular dependent measurements, the difference times the momentum to the fourth can be refined [29] and simultaneous refinement of the $I^{+}, I^{-}$reflectivity can be carried out. The magnetization can be described by an amplitude term and a specific orientation. The magnetic amplitude is refined by adjusting a weighting factor $w_{m}$ modifying the amplitude of the magnetic resonant terms. A value of one for $w_{m}$ corresponds to a magnetic moment that is derived from sum rules applied to the XMCD data. The formalism presented in the first part was coded [30]; we present an application in the following section.

\section{B. XRMR from a $\mathrm{W} / \mathrm{Fe} / \mathrm{W}$ trilayer}

We analyzed room temperature (RT) hard nonresonant and soft resonant magnetic reflectivity obtained at the $\mathrm{Fe} \mathrm{L}_{3}$-edge $(706.8 \mathrm{eV})$ for a trilayer $\mathrm{W} / \mathrm{Fe} / \mathrm{W}$. The multilayer was grown on $\mathrm{Al}_{2} \mathrm{O}_{3}$ by Pulsed Laser Deposition (the tungsten layers are used as a buffer and as a protection from oxidation). In order to determine the structural parameters, hard X-ray specular reflectivity shown in Fig. 6 was measured with a laboratory X-ray source $\left(\mathrm{Cu} \mathrm{K} \mathrm{K}_{\alpha}\right)$. The results of the refinement are summarized in Table $\mathrm{I}$.

The soft X-ray resonant magnetic scattering measurements were taken by switching the direction of a saturating applied magnetic field on U4B of the National Synchrotron Light Source (USA). The magnetic field (0.04

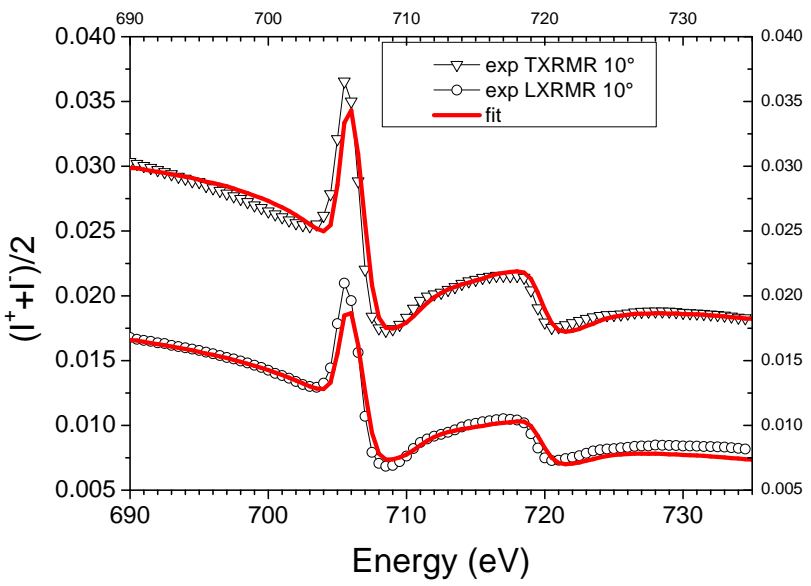

FIG. 7. Experimental (symbols) and calculated (solid line) of the specular reflectivity vs energy for an incident angle of 10 degrees. (LXRMR): applied field parallel to the surface, in the scattering plane, so-called longitudinal geometry, summing spectra for incident right and left circularly polarized light. (TXRMR): incident $\pi$-polarized light summing spectra from reversing a transverse field, applied parallel to the surface and perpendicular to the scattering plane (vertically offset by 0.01 for clarity).

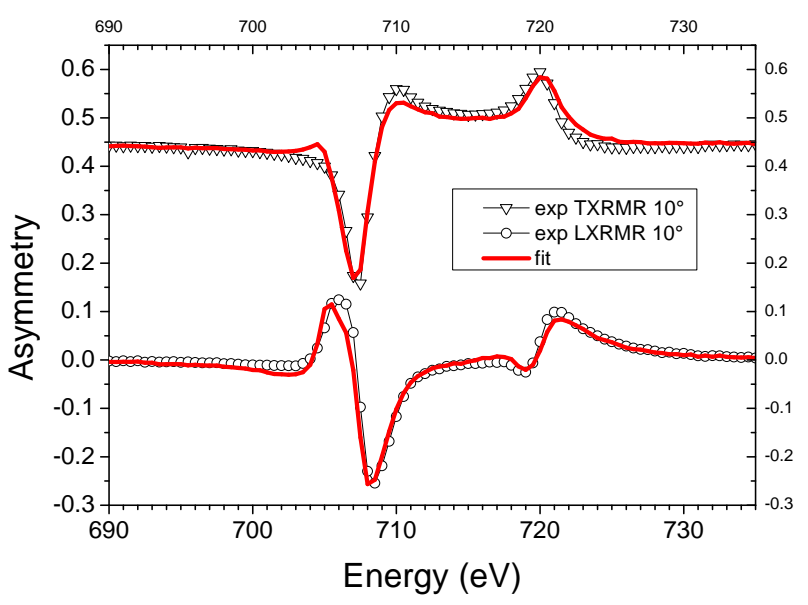

FIG. 8. Experimental (symbols) and calculated (solid line) asymmetry ratio $v s$ energy for an incident angle of 10 degrees for (open circle) circular polarized light and longitudinal applied field (LXRMR) and, (triangles) for $\pi$-polarized light and transverse applied field (TXRMR) (vertically offset by 0.45 for clarity).

TABLE I. Structural parameters obtained from the refinement of the hard X-ray reflectivity of the $\mathrm{W} / \mathrm{Fe} / \mathrm{W}$ trilayer.

\begin{tabular}{ccccc}
\hline layer & $d[\AA]$ & $\sigma[\AA]$ & $\rho\left[\mathrm{mol}_{\mathrm{cm}} \mathrm{cm}^{-3}\right]$ & $\rho\left[\mathrm{g} . \mathrm{cm}^{-3}\right]$ \\
\hline $\mathrm{W}$ & 31.3 & 1.4 & 0.105 & 19.30 \\
$\mathrm{Fe}$ & 91.5 & 5.1 & 0.141 & 7.875 \\
$\mathrm{~W}$ & 134.1 & 11.6 & 0.105 & 19.30 \\
$\mathrm{Al}_{2} \mathrm{O}_{3}$ & & 5.9 & 0.038 & 3.876 \\
\hline
\end{tabular}


T) was applied parallel to the surface of the sample, either in the scattering plane, the so called "longitudinal" configuration, or transverse to the scattering plane, the "transverse" configuration. In the first configuration, circular polarized light was used, and linear $\pi$ polarized light for the transverse configuration. Fig. 7 shows the dependence of the averaged reflectivity $\left(I^{+}+I^{-}\right) / 2$ against photon energy measured at 10 degrees for both configurations. The fits reproduce the resonant changes of intensity at the $\mathrm{L}_{3}$ and $\mathrm{L}_{2}$ edge as well as the variation apart from the resonance. For the simulations, the scattering factors for the iron at the L edges were obtained from the XMCD of Fe bcc which relates to the imaginary part [28], and a Kramers-Kronig transformation of the imaginary part to get the real part. Fig. 8 displays the asymmetry derived for both cases. The differences between the cases are ascribed to the different interplay between magnetization direction of the Fe layer and polarization state of the incident beam. The agreement between the fit and the experiment shows that the formalism can deal properly with different configurations.

Acknowledgements We thank Olivier Fruchart from the Institut Néel for lending us the sample $\mathrm{W} / \mathrm{Fe} / \mathrm{W}$. We acknowledge the use of the beamline U4B of the National Synchrotron Light Source, and thank the staff at U4B for their assistance.
[1] M. Faraday, Trans. R. Soc. (London), 5, 592 (1846).

[2] J. Kerr, Phil. Mag., 3, 339 (1877).

[3] Z. Qiu and S. Bader, Review of Scientific Instrument, 71, 1243 (2000).

[4] D. Gibbs, D. R. Harshman, E. D. Isaacs, D. B. McWhan, D. Mills, and C. Vettier, Phys. Rev. Lett., 61, 1241 (1988).

[5] J. P. Hannon, G. T. Trammell, M. Blume, and D. Gibbs, Phys. Rev. Lett., 61, 1245 (1988).

[6] V. B. Berestetskii, E. M. Lifshitz, and L. P. Pitaevskii, Relativistic Quantum Theory (New York: Pergamon Press, 1971).

[7] M. Blume, Resonant Anomalous X-Ray Scattering, Theory and Applications (Materlik, Sparks and Fisher, 1994).

[8] N. Stojic, N. Binggeli, and M. Altarelli, Physical Review B, 72, 104108 (2005).

[9] C. T. Chen, Y. U. Idzerda, H.-J. Lin, H. Smith, G. Meigs, E. Chaban, G. Ho, E. Pellegrin, and F. Sette, Phys. Rev. Lett., 75, 152 (1995).

[10] L. D. Landau, E. M. Lifshitz, and L. P. Pitaevskii, Electrodynamics of continuous media (Pergamon Press, 1984).

[11] L. Névot and P. Croce, Revue Phys. Appl., 15, 761 (1980).

[12] B. Vidal and P. Vincent, Applied Optics, 23, 1794 (1984).

[13] D. R. Lee, S. K. Sinha, D. Haskel, Y. Choi, J. C. Lang, S. A. Stepanov, and G. Srajer, Phys. Rev. B, 68, 224409 (2003).

[14] S. Valencia, A. Gaupp, W. Gudat, L. Abad, L. Balcells, and B. Martinez, Journal of Applied Physics, 104, 023903 (2008).

[15] M. Mansuripur, Journal of Applied Physics, 67 (1990).

[16] J. Zak, E. R. Moog, C. Liu, and S. D. Bader, Phys. Rev. B, 43, 6423 (1991).

[17] A. Bourzami, O. Lenoble, C. Féry, J. F. Bobo, and M. Piecuch, Phys. Rev. B, 59, 11489 (1999).

[18] M. Sacchi and A. Mirone, Phys. Rev. B, 57, 8408 (1998).
[19] S. A. Stepanov and S. K. Sinha, Phys. Rev. B, 61, 15302 (2000).

[20] C.-C. Kao, C. T. Chen, E. D. Johnson, J. B. Hastings, H. J. Lin, G. H. Ho, G. Meigs, J.-M. Brot, S. L. Hulbert, Y. U. Idzerda, and C. Vettier, Phys. Rev. B, 50, 9599 (1994).

[21] A. Déchelette, J. M. Tonnerre, M. C. S. Lager, F. Bartolomé, L. Sève, D. Raoux, H. Fischer, M. Piecuch, V. Chakarian, and C. C. Kao, Phys. Rev. B, 60, 6636 (1999).

[22] S. Roy, C. Sanchez-Hanke, S. Park, M. R. Fitzsimmons, Y. J. Tang, J. I. Hong, D. J. Smith, B. J. Taylor, X. Liu, M. B. Maple, A. E. Berkowitz, C.-C. Kao, and S. K. Sinha, Phys. Rev. B, 75, 014442 (2007).

[23] J. M. Tonnerre, M. D. Santis, S. Grenier, H. C. N. Tolentino, V. Langlais, E. Bontempi, M. Garcia-Fernandez, and U. Staub, Physical Review Letters, 100, 157202 (2008).

[24] H. L. Meyerheim, J.-M. Tonnerre, L. Sandratskii, H. C. N. Tolentino, M. Przybylski, Y. Gabi, F. Yildiz, X. L. Fu, E. Bontempi, S. Grenier, and J. Kirschner, Phys. Rev. Lett., 103, 267202 (2009).

[25] J. W. Freeland, J. Chakhalian, A. V. Boris, J.-M. Tonnerre, J. J. Kavich, P. Yordanov, S. Grenier, P. Zschack, E. Karapetrova, P. Popovich, H. N. Lee, and B. Keimer, Phys. Rev. B, 81, 094414 (2010).

[26] J. Geissler, E. Goering, M. Justen, F. Weigand, G. Schütz, J. Langer, D. Schmitz, H. Maletta, and R. Mattheis, Phys. Rev. B, 65, 020405 (2001).

[27] J. P. Hill and D. F. McMorrow, Acta Cryst., A52, 236 (1996).

[28] J. Als-Nielsen and D. McMorrow, Elements of Modern X-Ray Physics (Wiley, 2001).

[29] E. Kravtsov, D. Haskel, S. G. E. te Velthuis, J. S. Jiang, and B. J. Kirby, Phys. Rev. B, 79, 134438 (2009).

[30] The program uses Matlab environment and sources and binaries can be freely downloaded at http://neel.cnrs.fr/spip.php?rubrique553. 